\title{
Erratum: Intrinsic Defect Properties in Halide Double Perovskites for Optoelectronic Applications [Phys. Rev. Applied 10, 041001 (2018)]
}

\author{
Tianshu Li, ${ }^{1}$ Xingang Zhao, ${ }^{1}$ Dongwen Yang, ${ }^{1}$ Mao-Hua Du$\odot,{ }^{2}$ and Lijun Zhang ${ }^{1}$ \\ ${ }^{1}$ State Key Laboratory of Superhard Materials, Key Laboratory of Automobile Materials of MOE, and College of \\ Materials Science and Engineering, Jilin University, Changchun 130012, China \\ ${ }^{2}$ Materials Science and Technology Division, Oak Ridge National Laboratory, Oak Ridge, Tennessee 37831, USA
}

(Received 6 January 2021; published 26 January 2021)

DOI: 10.1103/PhysRevApplied.15.019901

There is an error in the calculated phase diagram of $\mathrm{Cs}_{2} \mathrm{AgInCl}_{6}$ in the paper "Intrinsic Defect Properties in Halide Double Perovskites for Optoelectronic Applications" [Phys. Rev. Applied 10, 041001 (2018)] [1]. The error is caused by the missing of a secondary phase of $\mathrm{Cs}_{3} \mathrm{In}_{2} \mathrm{Cl}_{9}$ (Fig. 2a). The corrected Fig. 2a is shown below.
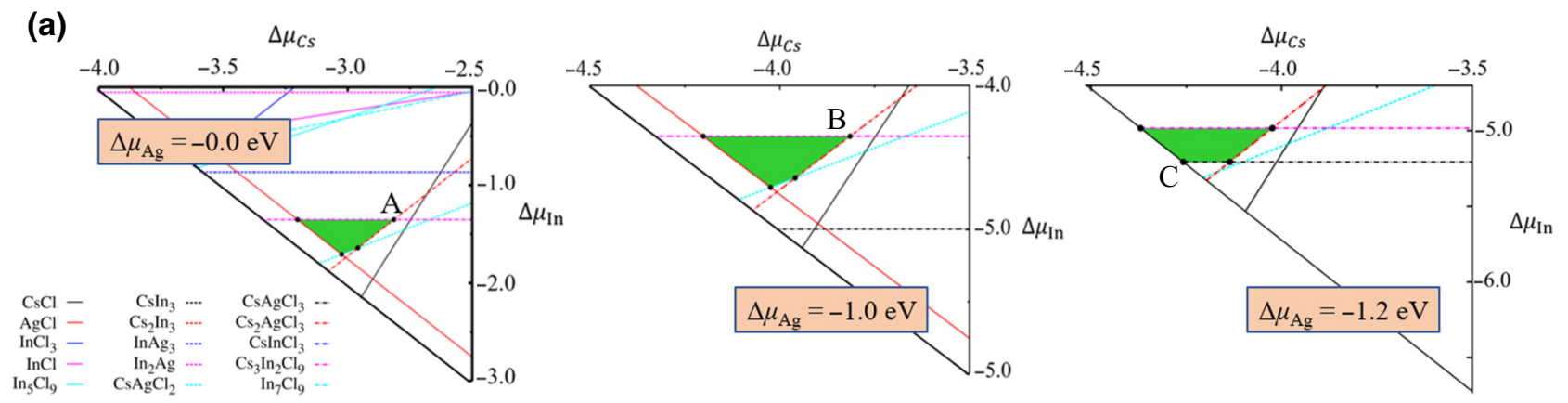

Corrected FIG. 2(a)

The inclusion of $\mathrm{Cs}_{3} \mathrm{In}_{2} \mathrm{Cl}_{9}$ as a competing phase to $\mathrm{Cs}_{2} \mathrm{AgInCl}_{6}$ lowers the maximum In chemical potential $\left(\Delta \mu_{\text {In }}\right)$ and increases the minimum $\mathrm{Ag}$ chemical potential $\left(\Delta \mu_{\mathrm{Ag}}\right)$ allowed for the growth of $\mathrm{Cs}_{2} \mathrm{AgInCl}_{6}$. As a result, the defect formation energies calculated at the In-rich limit [Fig. 3(a)-(b)] and at the Ag-poor limit (Fig. 3(c) and Fig. S1) need to be corrected as shown below. 

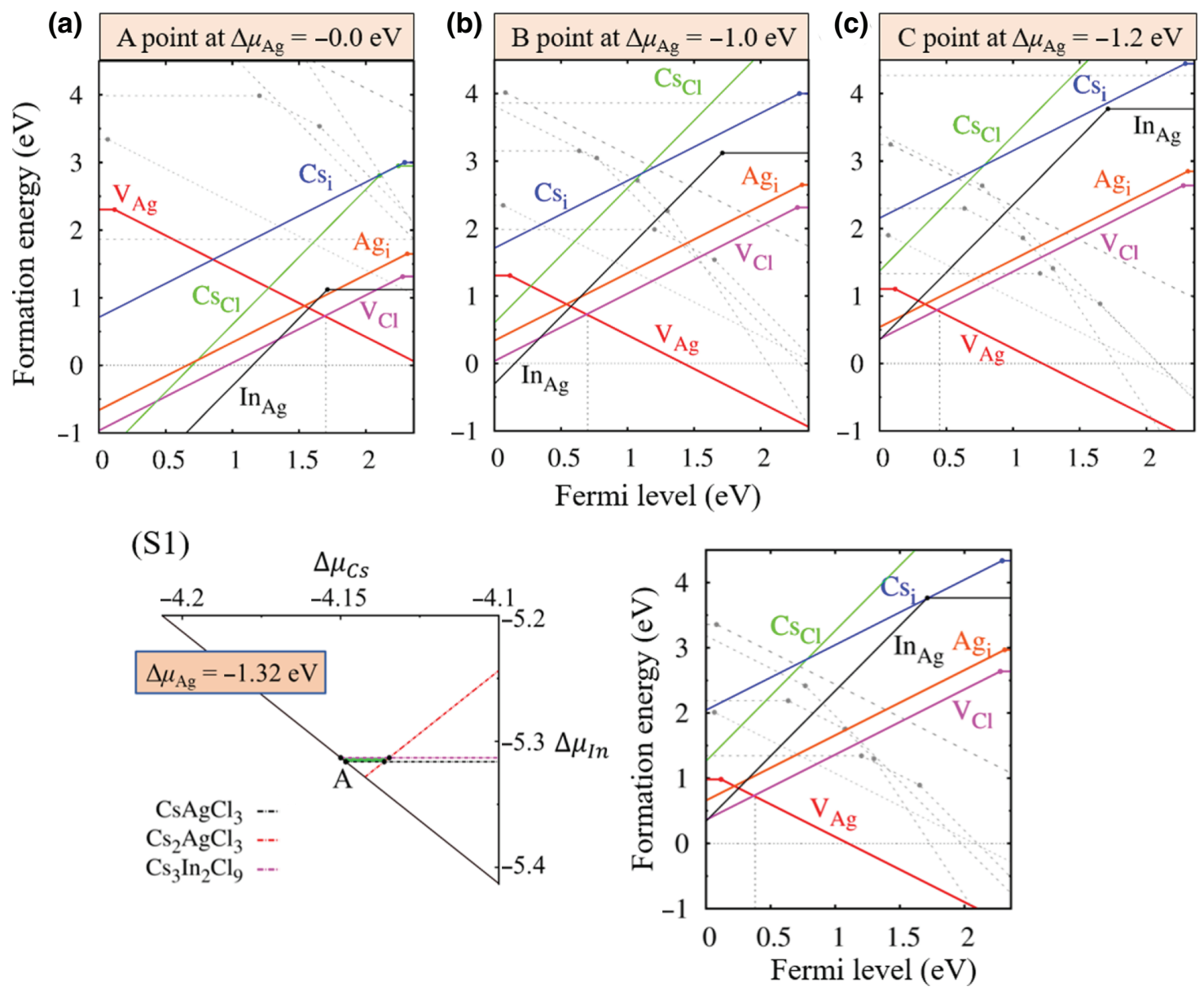

Corrected FIGs. 3(a-b) and S1.

The correction to the phase diagram leads to an increase of the calculated lowest-attainable Fermi level in $\mathrm{Cs}_{2} \mathrm{AgInCl}_{6}$, by about $0.1 \mathrm{eV}$ (the crossing point between $\mathrm{V}_{\mathrm{Cl}}$ and $\mathrm{V}_{\mathrm{Ag}}$ in Fig. $\mathrm{S} 1$ ), but does not affect the p-type conductivity because the Fermi level is still well below the midgap as shown in Fig. S1. The main conclusions of the paper remain valid.

We thank Prof. Zewen Xiao from Huazhong University of Science and Technology for pointing out this issue to us.

[1] T. Li, X. Zhao, D. Yang, M.-H. Du, and L. Zhang, Intrinsic Defect Properties in Halide Double Perovskites for Optoelectronic Applications, Phys. Rev. Appl. 10, 041001 (2018). 